\title{
A Bioinspired Neurodynamics-Based Approach to Tracking Control of Mobile Robots
}

\author{
Simon X. Yang, Senior Member, IEEE, Anmin Zhu, Member, IEEE, \\ Guangfeng Yuan, and Max Q.-H. Meng, Fellow, IEEE
}

\begin{abstract}
Tracking control is a fundamentally important issue for robot and motor systems, where smooth velocity commands are desirable for safe and effective operation. In this paper, a novel biologically inspired tracking control approach to real-time navigation of a nonholonomic mobile robot is proposed by integrating a backstepping technique and a neurodynamics model. The tracking control algorithm is derived from the error dynamics analysis of the mobile robot and the stability analysis of the closed-loop control system. The stability of the robot control system and the convergence of tracking errors to zeros are guaranteed by a Lyapunov stability theory. Unlike some existing tracking control methods for mobile robots whose control velocities suffer from velocity jumps, the proposed neurodynamics-based approach is capable of generating smooth continuous robot control signals with zero initial velocities. In addition, it can deal with situations with a very large tracking error. The effectiveness and efficiency of the proposed neurodynamics-based tracking control of mobile robots are demonstrated by experimental and comparison studies.
\end{abstract}

Index Terms-Backstepping control, Lyapunov stability, mobile robot, neural dynamics, tracking control.

\section{INTRODUCTION}

$\mathbf{R}$ EAL-TIME tracking control of robot and motor systems is a very important issue in robotics. The objective of path tracking of a robotic system is to generate robot velocities that drive the robot to track a desired path, i.e., the error between the desired path and the actual path is to converge to zero. Due to slippage, disturbance, noise, vehicle-terrain interaction, and sensor errors, it is very difficult to avoid the errors between the desired and actual robot paths. Thus, how to effectively and efficiently control a mobile robot to precisely track the desired robot paths is still an open challenging question in robotics.

Manuscript received December 18, 2009; revised July 28, 2010; accepted January 10, 2011. Date of publication March 22, 2011; date of current version March 30, 2012. This work was supported by the Natural Sciences and Engineering Research Council of Canada.

S. X. Yang is with the Advanced Robotics and Intelligent Systems Laboratory, School of Engineering, University of Guelph, Guelph, ON N1G 2W1, Canada (e-mail: syang@uoguelph.ca).

A. Zhu is with the School of Computer and Software, Shenzhen University, Shenzhen 518060, China, and also with the Advanced Robotics and Intelligent Systems Laboratory, University of Guelph, Guelph, ON N1G 1M8, Canada (e-mail: azhu@szu.edu.cn).

G. Yuan is with the Electrical Department, Detroit Heavy Truck Engineering, Novi, MI 48375 USA, and also with the Advanced Robotics and Intelligent Systems Laboratory, University of Guelph, Guelph, ON N1G 1M8, Canada (e-mail: gaviny@gmail.com).

M. Q.-H. Meng is with the Department of Electronic Engineering, Chinese University of Hong Kong, Hong Kong (e-mail: max@ee.cuhk.edu.hk).

Color versions of one or more of the figures in this paper are available online at http://ieeexplore.ieee.org.

Digital Object Identifier 10.1109/TIE.2011.2130491
There have been many studies on tracking control of robots or motors in recent years (e.g., [1]-[27]). The existing tracking control methods could be classified into four categories: 1) sliding mode [3], [7], [8], [17], [18], [20], [23], [26]-[29]; 2) linearization [4], [30]; 3) backstepping [2], [5], [12], [31], [32]; and 4) neural networks and fuzzy systems [1], [7], [11], [25], [29], [33], [34]. The control algorithms using the sliding mode technique are normally complicated and computationally expensive. The generated velocity commands with respect to time are not smooth curves, which may lead to discontinuousness in the robot velocities [3], [12]. The linearizationbased methods (e.g., [4] and [30]) are obtained by converting input-output nonlinear control systems into input-output linear control systems through the linearization of the static and dynamic state feedback (i.e., the decoupling matrix or transformation matrix). They require a small initial error between the desired and actual robot positions. In addition, the generated velocity normally changes sharply at the starting period. The backstepping-based tracking controllers (e.g., [2], [5], and [31]) are the most commonly used approach. They are very simple, and the system stability is guaranteed by a Lyapunov stability theory. In addition, some of the backstepping-based controllers can deal with arbitrarily large initial error. However, the generated robot velocity commands using the backstepping control approaches start with a very large value and suffer from velocity jumps when sudden tracking errors occur, i.e., the required accelerations and forces/torques are infinitely large at those velocity-jump points, which is not practically possible.

To resolve the impractical problem of large initial velocities which resulted from the backstepping technique, Fierro and Lewis [2] proposed a novel torque controller for a mobile robot by generating torque signals using computed torque control or a three-layer neural-network-based control. However, both control methods are computationally complicated. In addition, the computed torque control required the exact robot model that mostly is not available, while the neural network model requires online learning in order to make the robot perform properly, which is computationally expensive. Yang and $\mathrm{Hu}$ [35] proposed an efficient neural network approach for real-time torque control of a mobile robot with unknown robot dynamics, which uses a single-layer neural network by utilizing the robot regressor dynamics feature, instead of a three-layer neural network as in Fierro and Lewis' controller. However, an additional torque control algorithm is still needed to resolve the velocity jumps as the backstepping technique is used to generate the desired velocities [35]. Zhang et al. [5] proposed a controller based on backstepping and a neural network, where 
the backstepping technique is used for the tracking control, while the neural network is for compensating the robot dynamics. However, the mobile robot also starts with a very large initial velocity, and the algorithm is computationally expensive. The fuzzy-rule-based tracking control approaches (e.g., [10], [29], and [36]) can solve the problem of large initial robot velocities, but it is very difficult to formulate the fuzzy rules, which are usually obtained by trial and error based on human knowledge. The existing neural-network-based tracking control algorithms (e.g., [33], [37], and [38]) for a mobile robot require either online learning and/or offline training procedures before the controllers are capable of controlling the robot properly, which could be computational complicated and may be suitable for only certain trained situations.

This paper is aiming to directly tackle the impractical velocity-jump problem in the backstepping controller through analyzing the error dynamics and control design and developing a novel tracking controller that is capable of generating smooth and continuous velocity commands without relying on any torque control of the mobile robot. In this paper, inspired by the unique features of the neural dynamics models derived from Hodgkin and Huxley's membrane equation [39] for a biological neural system and based on the error dynamics analysis of the mobile robot, a novel tracking controller is proposed for realtime navigation of a nonholonomic mobile robot. The control signal consists of a component from a neural dynamics model and a component from the error dynamics that is similar to a proportional $(\mathrm{P})$ control. The stability of the control system and the asymptotical convergence of tracking errors to zero are rigorously proved using a Lyapunov stability analysis. Distinct from the previous neural-network-based approaches, no learning procedures are needed in the proposed control algorithm. The proposed controller is capable of generating smooth continuous control signals with zero initial robot velocities. In addition, it can deal with arbitrarily large tracking errors between the desired and current robot postures. The innovative use of bioinspired neurodynamics to tracking control of mobile robots resolves the impractical velocity-jump issue in the backstepping tracking control of mobile robots. The proposed neurodynamics-based tracking controller is simple yet efficient, as only one equation is added in comparison to the backstepping control technique. No torque controllers are required to resolve the velocity jumps.

Similar to many existing path tracking methods (e.g., [2], [4], [5], [28], and [31]), the proposed approach to real-time robot tracking control assumes that the desired robot paths are available through a real-time robot path planner and the current robot location is known through real-time multisensor fusion. In particular, it is assumed that the location of the robot is completely and accurately known through various sensor measurement, multisensor fusion, and signal processing systems. The autonomous navigation of mobile robots is very complicated and involves many challenging issues, such as real-time multisensor fusion, signal processing, path planning, tracking control, and torque control. The sensor placement and multisensor fusion are needed to obtain complete environment knowledge; the signal processing is required to remove sensor measurement and communication noise for accurate environ- mental information; the path planning is needed to generate real-time collision-free paths for robot navigation toward the targets; and the torque control is needed to generate control torque/force that drive the robot with the desired velocities. In indoor and closed environments, the complete knowledge of the workspace can be obtained through several charge-coupled device (CCD) cameras mounted over the ceiling and various onboard robot sensors. In outdoor and open environments, knowing the environment is a challenging task, which could be achieved through global positioning system, land marks, laser sensors, ultrasonic sensors, and various other sensors [40]. Related work on real-time path planning of mobile robots with limited onboard sensory information in known or unknown environments can be found in [41]-[43]. Knowing the accurate locations of the robot and obstacles in the environment at every time instant can be addressed using simultaneous map building/updating and localization methods [43], [44]. Realtime torque/acceleration control of mobile robots with unknown dynamics would be found in [2], [35], and [45]. This paper is to develop a novel tracking controller that is capable of generating smooth and continuous velocities that drive a mobile robot to track a path in real time, which is based on the preliminary work in [6].

This paper is organized as follows. In Section II, the background for the proposed approach is briefly introduced, including the nonholonomic mobile robot model, the tracking errors and error dynamics, the tracking control problem, and the neural dynamics model. The proposed neural-dynamicsbased tracking control algorithm for mobile robots and its stability and convergence analysis are presented in Section III. Section IV presents the simulation and experimental studies, including a mobile robot that is to track a straight line and a circular path. A comparison to the backstepping model is presented. Based on the similar concept of the proposed tracking controller, an extension for discrete paths is presented in Section V. Finally, some concluding remarks are summarized in Section VI.

\section{BACKGROUND}

In this section, the nonholonomic mobile robot model is first briefly presented. Then, the tracking error is defined, and the error dynamics is provided. After that, the tracking control problem is briefly stated. The biological membrane model and bioinspired shunting neural dynamics model are finally outlined.

\section{A. Nonholonomic Mobile Robot Model}

In a 2-D Cartesian workspace shown in Fig. 1, two coordinate systems are defined: the global coordinate system $\{X, O, Y\}$ and the local coordinate system $\{D, C, L\}$, where $D$ is the driving direction (longitudinal direction), $L$ is the lateral direction (latitudinal direction), and $C$ is the robot center point. The global coordinate system is fixed to the Cartesian workspace, and the local coordinate system is attached to the mobile platform. A posture of a nonholonomic mobile robot in the global coordinate system can be uniquely determined by 


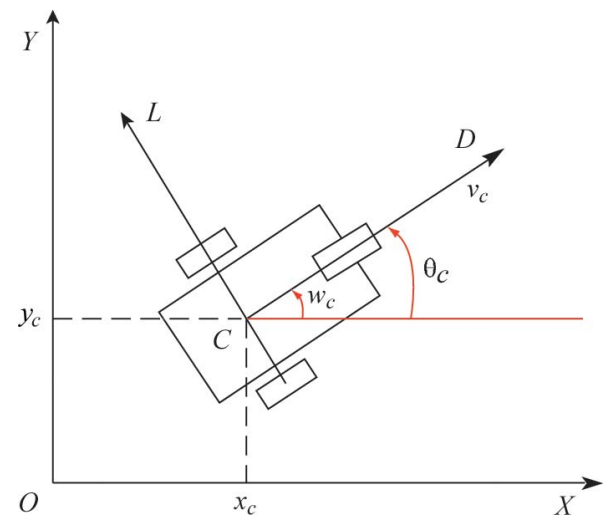

Fig. 1. Model of a nonholonomic mobile robot.

a vector $P_{c}=\left[x_{c}, y_{c}, \theta_{c}\right]^{T}$, where $\left[x_{c}, y_{c}\right]^{T}$ denotes the spatial position of the robot center $C$ and $\theta_{c}$ is the robot orientation angle with respect to point $C$ (see Fig. 1).

A freely movable mobile robot that is referred as a holonomic mobile robot has 3 degrees of freedom (DOF), $x_{c}, y_{c}$, and $\theta_{c}$. However, because of the kinematic constraint, the DOF for a nonholonomic mobile robot reduces to two. On the conditions of nonslipping, the kinematic constraint of a nonholonomic mobile robot is given as

$$
\dot{y}_{c} \cos \theta_{c}-\dot{x}_{c} \sin \theta_{c}=0
$$

From the motion control perspective, a mobile robot has $2 \mathrm{DOF}$, $v_{c}$ and $w_{c}$, where $v_{c}$ is the forward velocity (also called the linear velocity or the speed) and $w_{c}$ is the angular velocity of the mobile robot. For a nonholonomic mobile robot, the relationship between the velocity in the global coordinate system $\dot{P}_{c}=\left[\dot{x}_{c}, \dot{y}_{c}, \dot{\theta}_{c}\right]^{T}$ and the velocity $v=\left[v_{c}, w_{c}\right]^{T}$ in the local coordinate system can be described by a Jacobian matrix as

$$
\left[\begin{array}{l}
\dot{x}_{c} \\
\dot{y}_{c} \\
\dot{\theta}_{c}
\end{array}\right]=\dot{P}_{c}=\left[\begin{array}{cc}
\cos \theta_{c} & 0 \\
\sin \theta_{c} & 0 \\
0 & 1
\end{array}\right]\left[\begin{array}{l}
v_{c} \\
w_{c}
\end{array}\right] \text {. }
$$

\section{B. Tracking Error and Error Dynamics}

The reference path of a nonholonomic mobile robot provides the desired robot posture $P_{d}(t)=\left[x_{d}(t), y_{d}(t), \theta_{d}(t)\right]^{T}$ in the global coordinate system. The tracking error in the local coordinate system is defined as $E_{p}(t)=\left[e_{D}(t), e_{L}(t), e_{\theta}(t)\right]^{T}$, where $e_{D}, e_{L}$, and $e_{\theta}$ are the errors in the driving (longitudinal) direction, lateral direction, and orientation, respectively. The relationship between the tracking errors in the global and local coordinate systems can be obtained by geometrical projection transformation $T_{e}$ as

$$
E_{p}=\left[\begin{array}{c}
e_{D} \\
e_{L} \\
e_{\theta}
\end{array}\right]=\left[\begin{array}{ccc}
\cos \theta_{c} & \sin \theta_{c} & 0 \\
-\sin \theta_{c} & \cos \theta_{c} & 0 \\
0 & 0 & 1
\end{array}\right]\left[\begin{array}{c}
e_{x} \\
e_{y} \\
e_{\theta}
\end{array}\right]
$$

where $e_{x}=x_{d}-x_{c}, e_{y}=y_{d}-y_{c}$, and $e_{\theta}=\theta_{d}-\theta_{c}$ are the errors in the $X$ and $Y$ directions and orientation in the global coordinate system, respectively.
The error dynamics of the mobile robot can be derived from the time derivative of the aforementioned posture error equation in (3) as

$$
\left[\begin{array}{c}
\dot{e}_{D} \\
\dot{e}_{L} \\
\dot{e}_{\theta}
\end{array}\right]=\left[\begin{array}{c}
w_{c} e_{L}-v_{c}+v_{d} \cos e_{\theta} \\
-w_{c} e_{D}+v_{d} \sin e_{\theta} \\
w_{d}-w_{c}
\end{array}\right]
$$

where $v_{d}$ and $w_{d}$ are the desired forward velocity and angular velocity of the mobile robot, respectively.

\section{Tracking Control Problem}

The function of a tracking controller in this paper is to implement a mapping between the known information (e.g., the desired information and the sensory information) and the velocity commands designed to achieve the robot navigation task. The controller design problem can be described as follows. Given the desired robot posture $P_{d}(t)=\left[x_{d}(t), y_{d}(t), \theta_{d}(t)\right]^{T}$, i.e., the desired state of the mobile robot defined as

$$
X_{d}(t)=\left[x_{d}(t), y_{d}(t), \theta_{d}(t)\right]^{T}
$$

the desired forward and angular velocities can be deduced by

$$
\begin{aligned}
v_{d} & =\sqrt{{\dot{x_{d}}}^{2}+\dot{y}_{d}^{2}} \\
w_{d} & =\frac{\ddot{y_{d}} \dot{x_{d}}-\ddot{x_{d}} \dot{y_{d}}}{{\dot{x_{d}}}^{2}+\dot{y}_{d}{ }^{2}} .
\end{aligned}
$$

Then, design a control law for the forward velocity $v_{c}$ and angular velocity $w_{c}$ as a function of desired robot posture $P_{d}(t)$, which drive the robot to move, such that the actual robot state $X_{c}(t)$

$$
X_{c}(t)=\left[x_{c}(t), y_{c}(t), \theta_{c}(t)\right]^{T}
$$

precisely tracks the desired robot state $X_{d}(t)$, i.e.,

$$
\lim _{t \rightarrow \infty} X_{c}(t)=X_{d}(t) .
$$

In other words, the tracking error converges to zero as the time approaches infinite.

\section{Shunting Neural Dynamics Model}

Hodgkin and Huxley [39] proposed a model for a patch of membrane in a biological neural system using electrical circuit elements. This modeling work together with other experimental work led them to a Nobel Prize in 1963, for their discoveries concerning the ionic mechanisms involved in excitation and inhibition in the peripheral and central portions of the nerve cell membrane. In their membrane model, the dynamics of voltage across the membrane $V_{m}$ can be described using the state equation technique as

$$
\begin{aligned}
C_{m} \frac{d V_{m}}{d t}=-\left(E_{p}+V_{m}\right) g_{p}+\left(E_{N a}-\right. & \left.V_{m}\right) g_{N a} \\
& -\left(E_{K}+V_{m}\right) g_{K}
\end{aligned}
$$

where $C_{m}$ is the membrane capacitance. Parameters $E_{K}, E_{N a}$, and $E_{p}$ are the Nernst potentials (saturation potentials) for 
potassium ions, sodium ions, and the passive leak current in the membrane, respectively. Parameters $g_{K}, g_{N a}$, and $g_{p}$ represent the conductance of potassium, sodium, and passive channels, respectively. This model provided the foundation of the shunting model and led to a lot of model variations and applications [46]-[48].

By setting $C_{m}=1$ and substituting $x_{i}=E_{p}+V_{m}, A=g_{p}$, $B=E_{N a}+E_{p}, D=E_{k}-E_{p}, S_{i}^{+}=g_{N a}$ and $S_{i}^{-}=g_{K}$ in (10), a shunting equation is obtained [48]

$$
\frac{d x_{i}}{d t}=-A x_{i}+\left(B-x_{i}\right) S_{i}^{+}(t)-\left(D+x_{i}\right) S_{i}^{-}(t)
$$

where $x_{i}$ is the neural activity (membrane potential) of the $i$ th neuron. Parameters $A, B$, and $D$ are nonnegative constants representing the passive decay rate, the upper, and the lower bounds of the neural activity, respectively. Variables $S_{i}^{+}$and $S_{i}^{-}$ are the excitatory and inhibitory inputs to the neuron [48]. This shunting model was first proposed by Grossberg to understand the real-time adaptive behavior of individuals to complex and dynamic environmental contingencies [47], [49] and has a lot of applications in biological and machine vision, sensory motor control, and many other areas [47], [48]. Yang and Meng [50] developed the first innovative application to real-time robot path planning, which is later extended to various robotic systems [41], [43], [51]-[56] and further inspires the development of a distance-propagating system and a pulse coupled neural network for real-time path planning of mobile robots [57]-[59].

\section{Proposed Neurodynamics-Based Tracking CONTROL APPROACH}

The fundamental idea of the proposed approach is to incorporate a bioinspired neurodynamics component with the backstepping technique, where the sudden velocity jumps in the backstepping controllers are eliminated due to the smooth dynamics in the shunting neural model. In addition, Lyapunov stability analysis is used to prove the system stability and convergence of tracking errors to zero.

\section{A. Control Algorithm}

The tracking control law of a typical backstepping technique is given as

$$
\begin{aligned}
& v_{c}=c_{1} e_{D}+v_{d} \cos e_{\theta} \\
& w_{c}=w_{d}+c_{2} v_{d} e_{L}+c_{3} v_{d} \sin e_{\theta}
\end{aligned}
$$

where $c_{1}, c_{2}$, and $c_{3}$ are the parameters. By analyzing the backstepping-technique-based tracking controller used in [2], [5], [31], and [32], it is found that the velocity jumps are caused by the sudden changes in tracking errors, particularly the $e_{D}$, the position error along the longitudinal direction. Inspired by the smooth neural dynamics of the shunting neural models, a component $v_{s}$ characterized by a differential equation derived from a shunting model is used to replace the $e_{D}$ in the backstepping model in (12). Due to the dynamic behavior of $v_{s}$ as a function of $e_{D}$, the proposed control commands should become a smooth function of position errors. The output of the

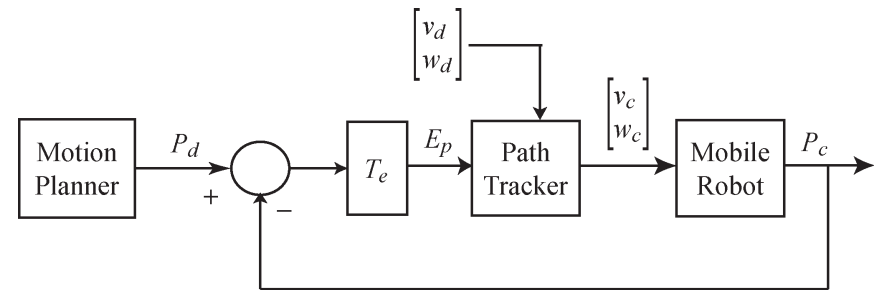

Fig. 2. System architecture of the proposed tracking control system.

shunting model changes as the input (tracking error) changes, smoothly without any jumps even when sudden sharp changes in the input occur. In addition, the output from the shunting model is bounded, and the transient response can be adjusted by its passive decay rate. Therefore, a neural dynamics component is incorporated in the proposed tracking controller.

The proposed tracking control law for the forward and angular velocities is given as

$$
\begin{aligned}
v_{c} & =v_{s}+v_{d} \cos e_{\theta} \\
w_{c} & =w_{d}+k_{2} v_{d} e_{L}+k_{3} v_{d} \sin e_{\theta}
\end{aligned}
$$

where $k_{2}$ and $k_{3}$ are positive parameters. The component $v_{s}$ is from a neural dynamics equation with respect to the error in the driving direction, which is given as

$$
\frac{d v_{s}}{d t}=-A v_{s}+\left(B-v_{s}\right) f\left(e_{D}\right)-\left(D+v_{s}\right) g\left(e_{D}\right)
$$

where $A$ is the passive decay rate of the velocity component and $B$ and $D$ are the upper bound and lower bound of the velocity component, respectively. Function $f(x)$ is a linearabove-threshold function defined as $f(x)=\max \{x, 0\}$, and the nonlinear function $g(x)$ is defined as $g(x)=\max \{-x, 0\}$.

At the start period, the velocity of the mobile robot should increase exponentially from zero to reach the desired velocity $v_{0}$. According to the time response property of the first-order system, the desired velocity can be defined as $v_{d}(t)=v_{0}(1-$ $\left.e^{-t / \tau}\right)$, where $v_{0}$ is the desired velocity and $\tau$ is the time constant.

The proposed tracking controller is used together with a path planner that generates real-time collision paths for the robot. The output of the path planner gives the desired robot posture $P_{d}(t)$. The current robot posture can be obtained through a robot localization algorithm using real-time multisensor fusion of various onboard sensor information and workspace monitoring information. The system architecture of the proposed tracking controller is shown in Fig. 2. The error vector $E_{p}$ in the local coordinate system is obtained through a transformation matrix $T_{e}$ using (3) from the posture error between the current and desired postures in the global coordinate system. The input of the path tracker is the error vector $E_{p}$ and the desired velocities $v_{d}$ and $w_{d}$. The path tracker generates the steering commands in forward velocity $v_{c}$ and angular velocity $w_{c}$ for the mobile robot. 


\section{B. Stability Analysis}

The tracking control system proposed in this paper is asymptotically stable, and the tracking errors converge to zeros. In the shunting (16), the excitatory input item $\left(B-v_{s}\right) f\left(e_{D}\right)$ forces the output of the shunting model to stay below the upper bound $B$ while the inhibitory input item $\left(D+v_{s}\right) g\left(e_{D}\right)$ guarantees that the output of the shunting model stays above the lower bound $-D$. Therefore, the output of the shunting model is bounded in the finite interval $[-D, B][52]$.

To rigorously prove the asymptotical stability and the error convergence of the proposed tracking control system, a Lyapunov function candidate is chosen as

$$
V(t)=\frac{1}{2}\left(e_{D}^{2}+e_{L}^{2}\right)+\frac{1}{k_{2}}\left(1-\cos e_{\theta}\right)+\frac{1}{2 B} v_{s}^{2}
$$

where $v_{s}$ is the velocity component defined in (16). It is obvious that $V(t) \geq 0$ and $V(t)=0$ if and only if $e_{D}=0, e_{L}=0$, $e_{\theta}=0$, and $v_{s}=0$.

From (4) and (14)-(16), the time derivative of the Lyapunov function becomes

$$
\begin{aligned}
\dot{V}(t)= & \dot{e}_{D} e_{D}+\dot{e}_{L} e_{L}+\frac{1}{k_{2}} \dot{e}_{\theta} \sin e_{\theta}+\frac{1}{B} \dot{v}_{s} v_{s} \\
= & \left(w_{c} e_{L}-v_{c}+v_{d} \cos e_{\theta}\right) e_{D} \\
& +\left(-w_{c} d_{D}+v_{d} \sin e_{\theta}\right) e_{L}+\frac{1}{k_{2}}\left(w_{d}-w_{c}\right) \\
& +\frac{1}{B}\left[-A v_{s}+\left(B-v_{s}\right) f\left(e_{D}\right)-\left(D+v_{s}\right) g\left(e_{D}\right)\right] \\
= & -v_{s} e_{D}-\frac{k_{3}}{k_{2}} v_{d} \sin ^{2} e_{\theta} \\
& +\frac{1}{B}\left[-A-f\left(e_{D}\right)-g\left(e_{D}\right)\right] v_{s}^{2} \\
& +\frac{1}{B}\left[B f\left(e_{D}\right)-D g\left(e_{D}\right)\right] v_{s} .
\end{aligned}
$$

If we choose the constants $B=D$ in the shunting equation, (18) can be rewritten as

$$
\begin{array}{r}
\dot{V}(t)=-\frac{k_{3}}{k_{2}} v_{d} \sin ^{2} e_{\theta}+\frac{1}{B}\left[-A-f\left(e_{D}\right)-g\left(e_{D}\right)\right] v_{s}^{2} \\
+\left[f\left(e_{D}\right)-g\left(e_{D}\right)-e_{D}\right] v_{s} .
\end{array}
$$

From the definition of $f\left(e_{D}\right)$ and $g\left(e_{D}\right)$, if $e_{D} \geq 0$, then $f\left(e_{D}\right)=e_{D}$ and $g\left(e_{D}\right)=0$. Thus, we have

$$
\left[f\left(e_{D}\right)-g\left(e_{D}\right)-e_{D}\right] v_{s}=\left[e_{D}-0-e_{D}\right]=0 .
$$

Similarly, if $e_{D}<0$, then $f\left(e_{D}\right)=0$ and $g\left(e_{D}\right)=-e_{D}$. Thus, we have

$$
\left[f\left(e_{D}\right)-g\left(e_{D}\right)-e_{D}\right] v_{s}=\left[0-\left(-e_{D}\right)-e_{D}\right]=0 .
$$

Therefore, (19) can be rewritten as

$$
\dot{V}(t)=-\frac{k_{3}}{k_{2}} v_{d} \sin ^{2} e_{\theta}+\frac{1}{B}\left[-A-f\left(e_{D}\right)-g\left(e_{D}\right)\right] v_{s}^{2} .
$$

Since parameters $k_{2}$ and $k_{3}$ are positive constants and the desired forward velocity $v_{d}$ is a positive constant, then we have $-\left(k_{3} / k_{2}\right) v_{d} \sin ^{2} e_{\theta} \leq 0$. From the definition of functions
$f\left(e_{D}\right)$ and $g\left(e_{D}\right)$, we have $f\left(e_{D}\right) \geq 0$ and $g\left(e_{D}\right) \geq 0$. In addition, the parameters $A$ and $B$ are nonnegative constants; thus, we have $\left[-A-f\left(e_{D}\right)-g\left(e_{D}\right)\right] v_{s}^{2} / B \leq 0$. Therefore, the time derivative of the Lyapunov function candidate $\dot{V}(t)$ in (22) along all the system trajectories is not larger than zero, i.e.,

$$
\dot{V}(t) \leq 0
$$

Thus, the proposed tracking control system for a mobile robot is stable.

The velocity error dynamic equations for a mobile robot satisfy the following relationship: $v_{c} \rightarrow v_{d}, w_{c} \rightarrow w_{d}$ as $t \rightarrow$ $\infty$. Obviously, by assuming that $v_{d}>0, \dot{V}(t) \leq 0$ and the entire error $E_{p}$ and $v_{s}$ are bounded. From (4) and $\dot{V}(t) \leq 0$, it can be inferred that $\left\|E_{p}\right\|,\left\|\dot{E}_{p}\right\|,\left\|v_{s}\right\|$, and $\left\|\dot{v}_{s}\right\|$ are bounded. Thus, we have $\|\ddot{V}(t)\|<\infty$ since $V(t)$ does not increase and converges to a certain constant value. By Barbalat's lemma, $\dot{V}(t) \rightarrow 0$ as $t \rightarrow \infty$, from which it can be deduced that $v_{s} \rightarrow 0$ as $t \rightarrow \infty$. By using (16) and the input-output property of the shunting model, it can be inferred that, if the output converges to some constant value (zero), the input is supposed to go to a constant value (zero), namely, $e_{D} \rightarrow 0$ as $v_{s} \rightarrow 0$. From the first term in (22), $\left(k_{3} / k_{2}\right) v_{d} \sin ^{2} e_{\theta}=0$, we have $e_{\theta} \rightarrow 0$ as $t \rightarrow$ $\infty$. From (4), as $e_{\theta} \rightarrow 0$, we have $w_{d}-w_{c}=0$. From (15), $e_{w} \rightarrow 0$ as $t \rightarrow \infty$; thus, $k_{2} v_{d} e_{L}=0$. From the assumption $v_{d}>0$, we have $e_{L} \rightarrow 0$ as $t \rightarrow \infty$. Thus, the equilibrium point is $E_{p}=0$. Therefore, the tracking control system is asymptotically stable. From the Jacobean transformation in (2), it is obvious that $x_{d}-x_{c}=0$ and $y_{d}-y_{c}=0$ from the results $e_{D}=0$ and $e_{L}=0$. Therefore, it has

$$
\lim _{t \rightarrow \infty}\left(X_{d}(t)-X_{c}(t)\right)=0
$$

That is, the proposed control algorithm is asymptotically stable, and the tracking errors are guaranteed to converge to zeros.

\section{Simulation and Experimental Studies}

In this section, the proposed tracking controller for a mobile robot is first applied to the tracking of a straight path and a circular path. After that, a comparison study to the backstepping model is presented. Finally, the proposed tracking control algorithm is applied to a real mobile robot in our research laboratory.

\section{A. Tracking a Straight Path}

To illustrate the effectiveness of the proposed approach, a simple straight line is first used as the desired robot path, which is described as $y=5$ and $x=0$ in the Cartesian workspace. The robot starts at posture $(0,3.2,0)$, while the desired initial robot posture is $(0,5,0)$. Thus the initial posture error is $(0,1.8$, $0)$. Time varies from 0 to $10 \mathrm{~s}$. The model parameters are chosen as follows: $k_{2}=5, k_{3}=2, A=5, B=3, D=3, v_{d}=1$, and $w_{d}=0$. During the initial period, the parameters used in the first-order exponential equation for the forward velocity $v_{d}$ are chosen as $k=1$ and $\tau=0.5$. The sampling time is $0.01 \mathrm{~s}$.

The performance of the mobile robot is shown in Fig. 3(a), where the actual robot path is denoted by the dash-dot line 

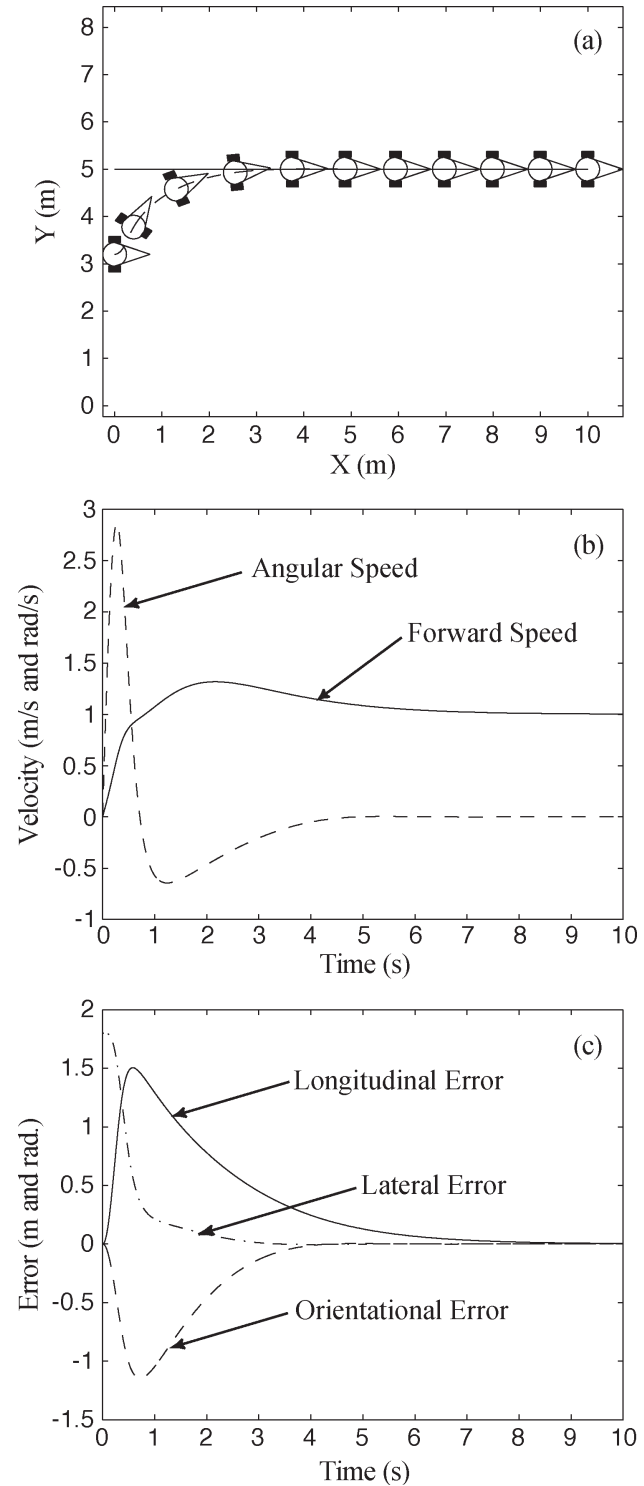

Fig. 3. Tracking a straight path. (a) Dynamic tracking performance. (b) Generated forward and angular velocities. (c) Tracking errors in the longitudinal and lateral directions and in orientation.

and the desired robot path is the solid line. The forward and angular velocities using the proposed tracking controller are shown in Fig. 3(b). It shows that the velocity commands are smooth and continuous without any jumps. The error dynamics in longitude, lateral, and orientation are shown in Fig. 3(c), which is converging to zero as time increases.

\section{B. Tracking a Circular Path}

Then, a circular path, $x^{2}+y^{2}=1$, shown in Fig. 4, is used in the simulation study. Assume that the desired forward and angular velocities are 1 and 1, respectively. The desired robot starts at such velocities from the initial posture $(1,0,0)$. The actual robot starts at posture $(0,-0.6,0)$, which means that the initial error is $(-1,-0.6,0)$. During the beginning period, the parameters used in the first-order exponential equation for forward velocity are $k=1$ and $\tau=0.05$. The parameters used in the controller are $A=5, B=3, D=3, K_{2}=2$, and
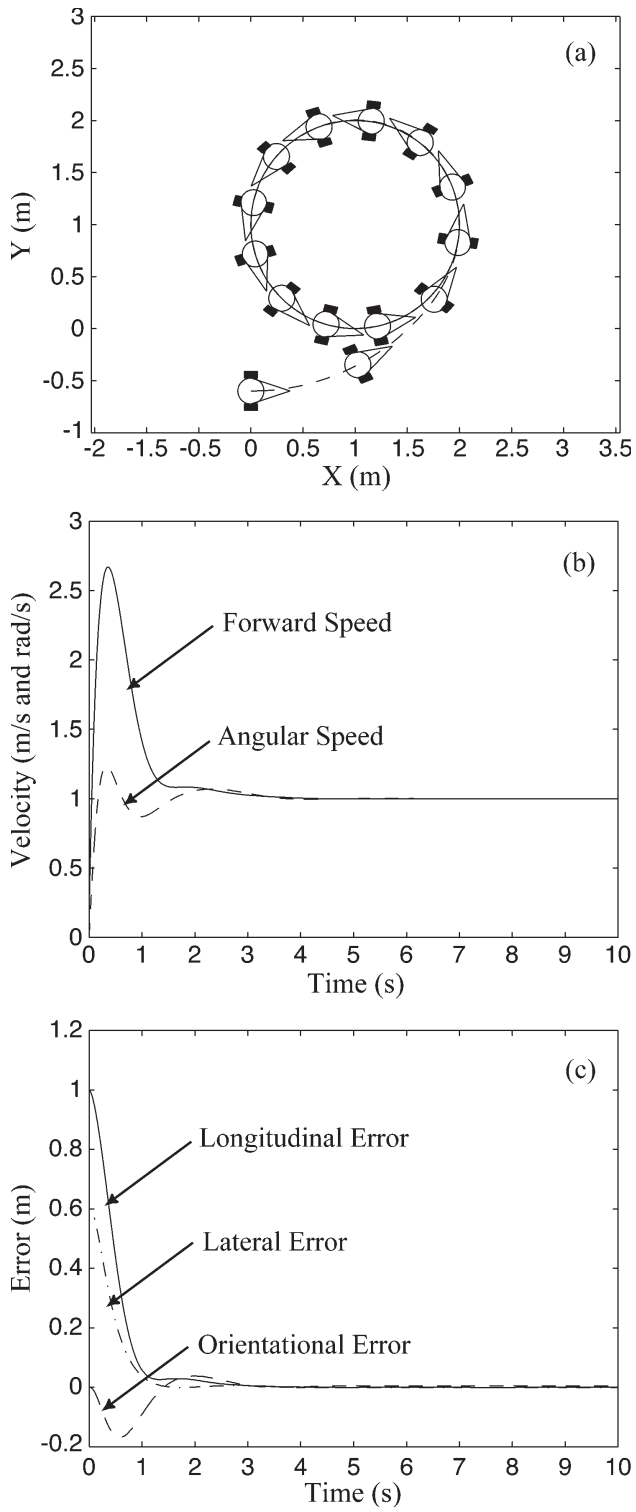

Fig. 4. Tracking a circular path. (a) Dynamic tracking performance. (b) Generated forward and angular velocities. (c) Tracking errors in the longitudinal and lateral directions and in orientation.

$k_{3}=4$. The sampling time is $0.01 \mathrm{~s}$. The performance of the mobile robot is shown in Fig. 4(a). It shows that the mobile robot chooses a perfect angular to land onto the desired circular path. Fig. 4(b) shows the smooth and reasonable velocity commands. The tracking errors are shown in Fig. 4(c), which are approaching to zero as time increases.

\section{Comparison to the Backstepping Controller}

To demonstrate the effectiveness of the proposed tracking controller, a comparison study to the backstepping controller is conducted. The tracking control law defined in (12) and (13) is used in our simulation study under the same condition as that in Fig. 4. The model parameters are chosen as the same as the proposed controller, i.e., $c_{1}=10, c_{2}=2$, and $c_{3}=4$. The performance is shown in Fig. 5(a). It shows that it takes a longer time for the robot to land on the desired circular path. The forward and angular robot velocities generated by the 

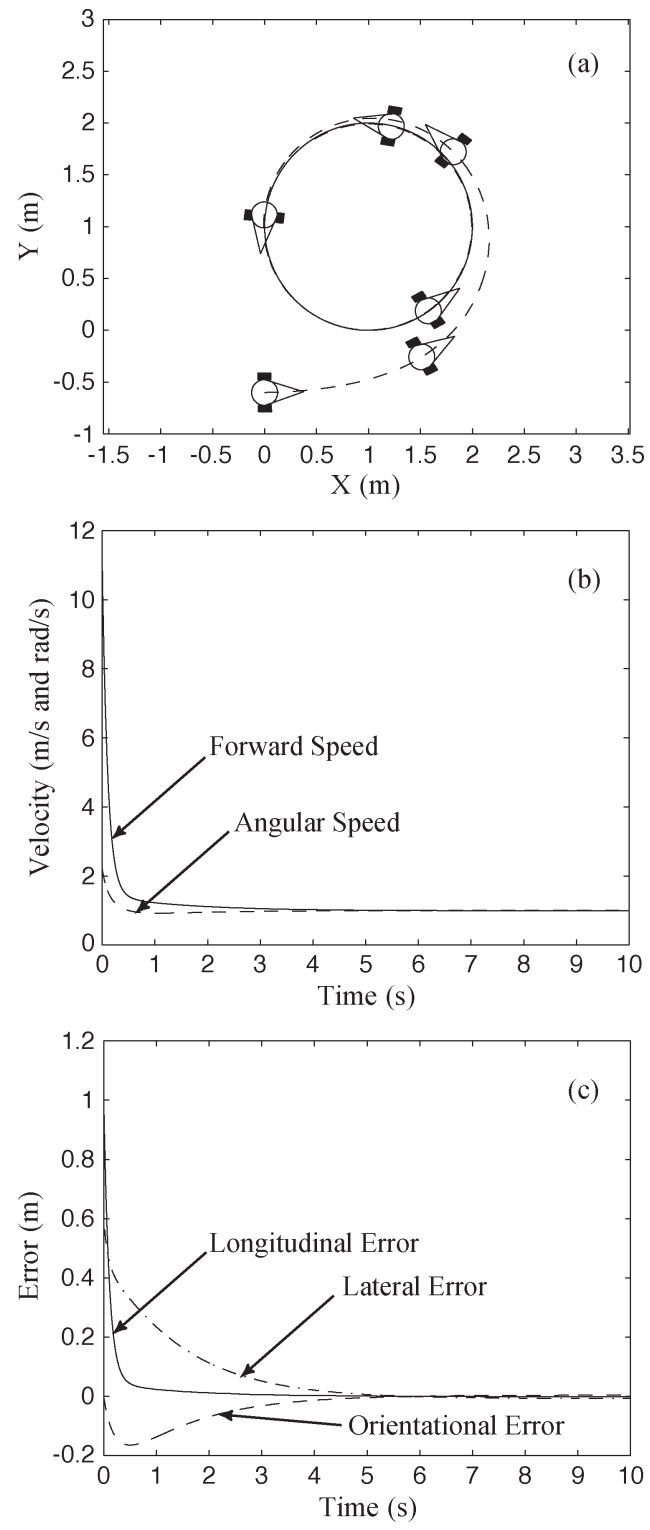

Fig. 5. Tracking a circular path using the backstepping controller. (a) Dynamic tracking performance. (b) Generated forward and angular velocities. (c) Tracking errors in the longitudinal and lateral directions and in orientation.

backstepping tracking controller are shown in Fig. 5(b). It shows that the velocity changes suddenly rather than gradually from zero at the initial time $t=0$. This implies that the forward and angular accelerations are infinitely large at the beginning, and it also implies that the robot force/torque should be infinitely large as well, which is not feasible in practice. In comparison to the results in Fig. 4(b) using the proposed tracking controller, both the forward and angular velocity commands are smooth and reasonable. In addition, the proposed controller tracks the circular path faster than the backstepping controller.

\section{Experiments on a Real Mobile Robot}

To validate the proposed neural-dynamics-based tracking controller for a mobile robot, experiments have been conducted using the mobile robot shown in Fig. 6 in our research laboratory. This mobile robot is equipped with a CCD camera as its

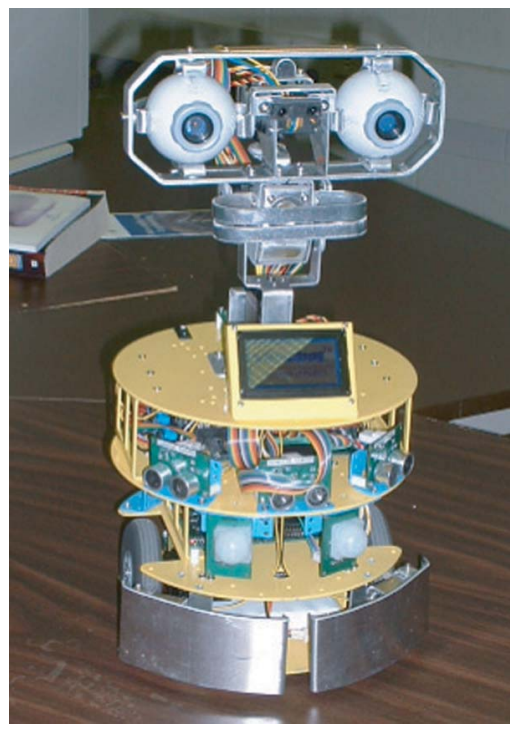

Fig. 6. Mobile robot used to conduct experiments of the proposed tracking controller.

vision system, five ultrasonic sensors to detect obstacles near the robot, and three bump sensors to stop the robot once it hits on any obstacles. The robot runs on a wheel-based platform with rotary sensors (odometer) mounted on each wheel to precisely measure the wheel movement. This robot is used to track both the straight path and circular path. In the experiments, the camera is used to obtain the desired location (in the experiment, a red object is moving along the desired path, providing the desired robot postures $\left.P_{d}(t)\right)$. The sensory information from the wheels is used to calculate the current robot posture $P_{c}(t)$. The two wheels of the mobile robot are controlled by

$$
\begin{aligned}
& w_{L}=\frac{1}{R}\left(v_{c}-\frac{D_{w}}{2} w_{c}\right) \\
& w_{R}=\frac{1}{R}\left(v_{c}+\frac{D_{w}}{2} w_{c}\right)
\end{aligned}
$$

where $w_{L}$ and $w_{R}$ are the angular velocities of the left and right wheels, respectively; $R$ is the radius of the wheels; and $D_{w}$ is the azimuth length between the two driven wheels [60]. The forward and angular velocities, $v_{c}$ and $w_{c}$, generated by the tracking control algorithms in Section II are used to calculate the wheel angular velocities. The experimental results are in agreement with the simulation results.

\section{Discussion}

The proposed tracking control algorithm in Section II is capable of generating smooth and continuous command for a mobile robot to track any smooth robot trajectories. In some situations, the desired robot paths generated by some robot path planners may be discrete, and many real roads in cities are basically joined with a number of discrete segments. Similar to the analysis of position error $e_{D}$ that results in velocity jumps, for discrete paths, the orientation error $e_{\theta}$ in (13) and (15) will also result in velocity jumps in both forward and angular velocities. 
Based on the same concept in Section II, the proposed algorithm can be extended by adding another shunting neural model to replace $e_{\theta}$. Thus, the neural-dynamics-based controller for the discrete path is given as

$$
\begin{aligned}
v_{c} & =v_{s}+v_{r} \cos \beta, \\
w_{c} & =w_{d}+k_{2} v_{r} e_{L}+k_{3} v_{d} \sin \beta
\end{aligned}
$$

where the two components $v_{s}$ and $\beta$ are characterized by two shunting neural models as

$$
\begin{aligned}
\frac{d v_{s}}{d t} & =-A_{c} v_{s}+\left(B_{c}-v_{s}\right) f\left(e_{D}\right)-\left(D_{c}+v_{s}\right) g\left(e_{D}\right) \\
\frac{d \beta}{d t} & =-A_{c} \beta+\left(B_{c}-\beta\right) f\left(e_{\theta}\right)-\left(D_{c}+\beta\right) g\left(e_{\theta}\right)
\end{aligned}
$$

where $A_{c}, B_{c}$, and $D_{c}$ are positive constants. In most backstepping-based models such as those in [2], [5], [31], and [32], the desired forward velocity $v_{d}$ is defined as a constant $v_{0}$, while the desired angular velocity $w_{d}$ is defined as zero. In order to obtain smooth velocity commands at the stop as well, $v_{d}$ at the stop is defined as $v_{d}=v_{0}\left(1-e^{s / \tau_{2}}\right)$, where $\tau_{2}$ is the time constant and $s$ is the distance from the current robot position to the stop position.

To demonstrate the effectiveness of the extended tracking controller for discrete paths, the tracking control algorithm in (27) and (28) is applied to a simple discretized path, with a comparison to the backstepping controller described in (12) and (13). The desired robot path is shown in Fig. 7(a), where the starting and target postures are $(5,5,4)$ and $(10,15,5)$, respectively. The desired forward and angular velocities are chosen as $v_{d}=1$ and $w_{d}=0$, respectively. The control parameters are chosen as follows: $A_{c}=10, B_{c}=D_{c}=3, c_{2}=c_{3}=2$, $v_{0}=1$, and $\tau=\tau_{2}=0.5$. The generated command signals using the proposed controller for forward velocity (solid line) and angular velocity (dashed line) are shown in Fig. 7(b), and the tracking errors in the longitude direction, lateral direction, and orientation are shown in Fig. 7(c). For comparison, the generated control commands using the backstepping controller are shown in Fig. 7(d). It is obvious that the proposed controller is capable of generating smooth continuous velocity commands [see Fig. 7(b)]. The control signals generated by the backstepping controller suffer from sharp jumps in both forward and angular velocities [see Fig. 7(d)], which implies that the corresponding accelerations and torques/forces are infinitely large at those velocity jumps when using the backstepping controller.

\section{CONCLUSION}

In this paper, a novel bioinspired neurodynamics-based tracking controller has been proposed by incorporating the biologically inspired neural dynamics model into the backstepping technique. The proposed tracking controller is capable of generating smooth and continuous velocity commands with zero initial value. The tracking control system is stable, and the tracking errors are guaranteed to converge to zeros. An extension for discrete paths is discussed, which does not suffer
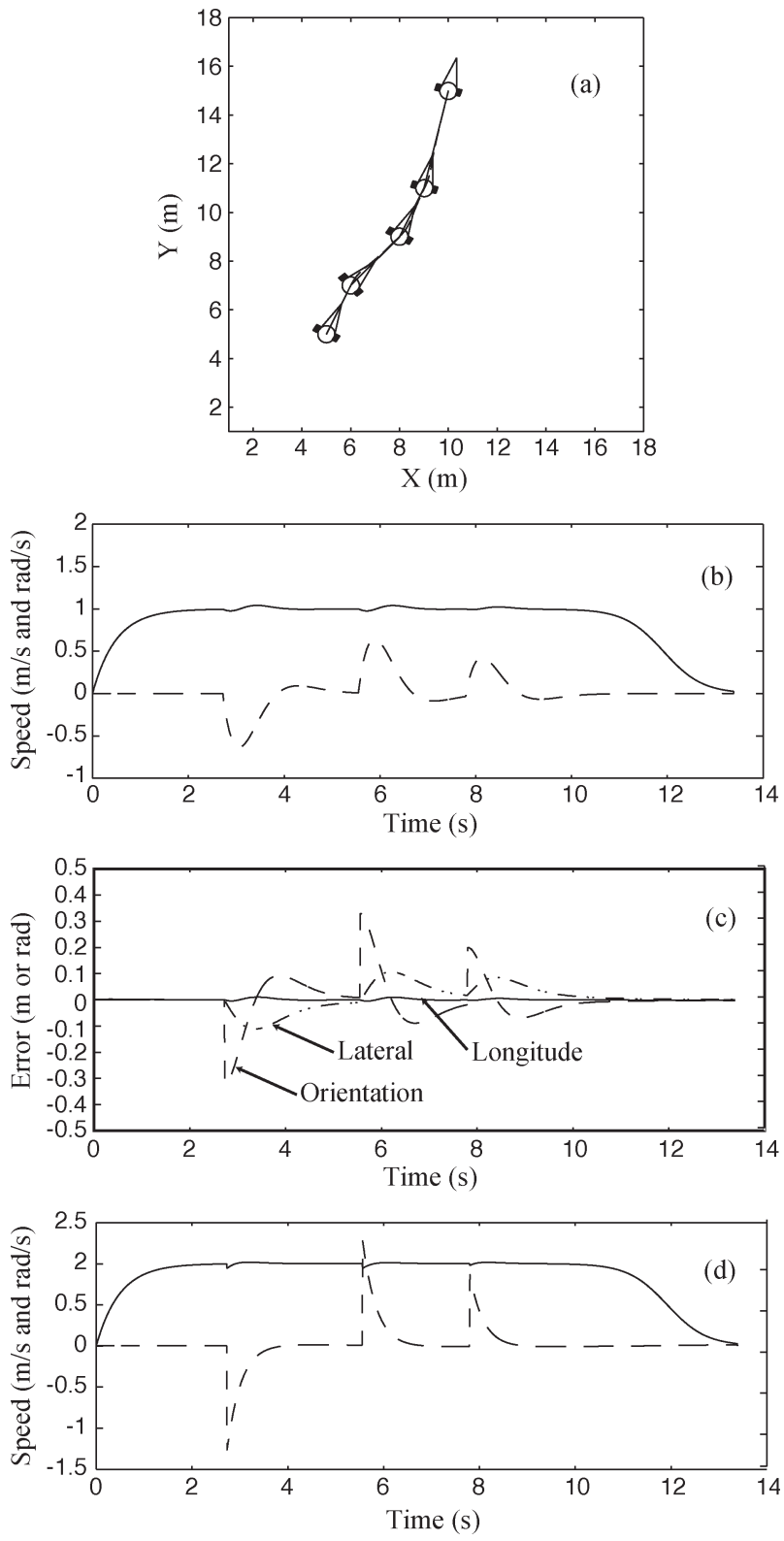

Fig. 7. Tracking control with a simple discretized path using the proposed controller and the backstepping controller. (a) Desired robot path. (b) Generated forward and angular velocity commands using the proposed controller. (c) Tracking errors in the longitude and lateral directions and in the orientation using the proposed controller. (d) Generated forward and angular velocity commands using the backstepping controller.

from both forward and angular velocity jumps existing in the backstepping controller.

\section{ACKNOWLEDGMENT}

The authors would like to thank the associate editor and anonymous reviewers for their valuable comments.

\section{REFERENCES}

[1] H.-M. Tai, J. Wang, and K. Ashenayi, "A neural network-based tracking control system," IEEE Trans. Ind. Electron., vol. 39, no. 6, pp. 504-510, Dec. 1992.

[2] R. Fierro and F. V. Lewis, "Control of a nonholonomic mobile robot: Backstepping kinematics into dynamics," J. Robot. Syst., vol. 14, no. 3, pp. 149-163, 1997. 
[3] J. M. Yang and J. H. Kim, "Sliding mode control for trajectory tracking of nonholonomic wheeled mobile robots," IEEE Trans. Robot. Autom., vol. 15 , no. 3, pp. 578-587, Jun. 1999.

[4] D. H. Kim and J. H. Oh, "Tracking control of a two-wheeled mobile robot using input-output linearization," Control Eng. Practice, vol. 7, no. 3, pp. 369-373, Mar. 1999.

[5] Q. Zhang, J. Shippen, and B. Jones, "Robust backstepping and neural network control of a low-quality nonholonomic mobile robot," Int. J. Mach. Tools Manuf., vol. 39, no. 7, pp. 1117-1134, Jul. 1999.

[6] G. Yuan, S. X. Yang, and G. S. Mittal, "Tracking control of a mobile robot using a neural dynamics based approach," in Proc. IEEE Int. Conf. Robot. Autom., Seoul, Korea, May 2001, vol. 1, pp. 163-168.

[7] R.-J. Wai and K.-H. Su, "Adaptive enhanced fuzzy sliding-mode control for electrical servo drive," IEEE Trans. Ind. Electron., vol. 53, no. 2, pp. 569-580, Apr. 2006.

[8] R.-J. Wai and L.-J. Chang, "Adaptive stabilizing and tracking control for a nonlinear inverted-pendulum system via sliding-mode technique," IEEE Trans. Ind. Electron., vol. 53, no. 2, pp. 674-692, Apr. 2006.

[9] A. Zhu and S. X. Yang, "Real-time tracking control of a nonholonomic mobile robot with bounded accelerations," Int. J. Veh. Auton. Syst., vol. 14, no. $2 / 3$, pp. 156-172, Jan. 2007

[10] G. Antonelli, S. Chiaverini, and G. Fusco, "A fuzzy-logic-based approach for mobile robot path tracking," IEEE Trans. Fuzzy Syst., vol. 15, no. 2, pp. 211-221, Apr. 2007.

[11] R.-J. Wai, "Fuzzy sliding-mode control using adaptive tuning technique," IEEE Trans. Ind. Electron., vol. 54, no. 1, pp. 586-594, Feb. 2007.

[12] H. Ashrafiuon, K. R. Muske, L. C. McNinch, and R. A. Soltan, "Slidingmode tracking control of surface vessels," IEEE Trans. Ind. Electron., vol. 55, no. 11, pp. 4004-4012, Nov. 2008.

[13] H.-H. Lin, C.-C. Tsai, and J.-C. Hsu, "Ultrasonic localization and pose tracking of an autonomous mobile robot via fuzzy adaptive extended information filtering," IEEE Trans. Instrum. Meas., vol. 57, no. 9, pp. 20242034, Sep. 2008.

[14] C. B. Low and D. Wang, "GPS-based tracking control for a car-like wheeled mobile robot with skidding and slipping," IEEE/ASME Trans. Mechatronics, vol. 13, no. 4, pp. 480-484, Aug. 2008.

[15] K. Do, "Formation tracking control of unicycle-type mobile robots with limited sensing ranges," IEEE Trans. Control Syst. Technol., vol. 16, no. 3, pp. 527-538, May 2008

[16] M. Jin, S. H. Kang, and P. H. Chang, "Robust compliant motion control of robot with nonlinear friction using time-delay estimation," IEEE Trans. Ind. Electron., vol. 55, no. 1, pp. 258-269, Jan. 2008.

[17] M. Defoort and T. Murakami, "Sliding-mode control scheme for an intelligent bicycle," IEEE Trans. Ind. Electron., vol. 56, no. 9, pp. 3357-3368, Sep. 2009.

[18] M.-S. Park and D. Chwa, "Swing-up and stabilization control of invertedpendulum systems via coupled sliding-mode control method," IEEE Trans. Ind. Electron., vol. 56, no. 9, pp. 3541-3555, Sep. 2009.

[19] N. Uchiyama, T. Hashimoto, S. Sano, and S. Takagi, "Model-reference control approach to obstacle avoidance for a human-operated mobile robot," IEEE Trans. Ind. Electron., vol. 56, no. 10, pp. 3892-3896, Oct. 2009

[20] K. Abidi, J.-X. Xu, and J.-H. She, "A discrete-time terminal sliding-mode control approach applied to a motion control problem," IEEE Trans. Ind. Electron., vol. 56, no. 9, pp. 3619-3627, Sep. 2009.

[21] H. Chen, M.-M. Ma, H. Wang, Z.-Y. Liu, and Z.-X. Cai, "Moving horizon $\mathcal{H}_{\infty}$ tracking control of wheeled mobile robots with actuator saturation," IEEE Trans. Control Syst. Technol., vol. 17, no. 2, pp. 449-457, Mar. 2009.

[22] C.-Y. Tsai and K.-T. Song, "Visual tracking control of a wheeled mobile robot with system model and velocity quantization robustness," IEEE Trans. Control Syst. Technol., vol. 17, no. 3, pp. 520-527, May 2009.

[23] M. Jin, J. Lee, P. H. Chang, and C. Choi, "Practical nonsingular terminal sliding-mode control of robot manipulators for high-accuracy tracking control," IEEE Trans. Ind. Electron., vol. 56, no. 9, pp. 3593-3601, Sep. 2009.

[24] K. Saiki, A. Hara, K. Sakata, and H. Fujimoto, "A study on high-speed and high-precision tracking control of large-scale stage using perfect tracking control method based on multirate feedforward control," IEEE Trans. Ind. Electron., vol. 57, no. 4, pp. 1393-1400, Apr. 2010.

[25] C.-C. Tsai, H.-C. Huang, and S.-C. Lin, "Adaptive neural network control of self-balancing two-wheeled scooter," IEEE Trans. Ind. Electron., vol. 57, no. 4, pp. 1420-1428, Apr. 2010.

[26] J. Zhang and Y. Xia, "Design of static output feedback sliding mode control for uncertain linear systems," IEEE Trans. Ind. Electron., vol. 57, no. 6, pp. 2161-2170, Jun. 2010.
[27] C. J. Fallaha, M. Saad, H. Y. Kanaan, and K. Al-Haddad, "Sliding-mode robot control with exponential reaching law," IEEE Trans. Ind. Electron., vol. 58, no. 2, pp. 600-610, Feb. 2011.

[28] D. Chwa, "Sliding-mode tracking control of nonholonomic wheeled mobile robots in polar coordinates," IEEE Trans. Control Syst. Technol., vol. 12, no. 4, pp. 637-644, Jul. 2004.

[29] G. G. Rigatos, C. S. Tzafestas, and S. G. Tzafestas, "Mobile robot motion control in partially unknown environments using a sliding-mode fuzzylogic controller," Robot. Auton. Syst., vol. 33, no. 1, pp. 1-11, Oct. 2000.

[30] D. J. Leith and W. E. Leithead, "Gain-scheduled and nonlinear systems: Dynamic analysis by velocity-based linearization families," Int. J. Control, vol. 70, no. 2, pp. 289-317, May 1998.

[31] P.-S. Tsai, L.-S. Wang, F.-R. Chang, and T.-F. Wu, "Systematic backstepping design for b-spline trajectory tracking control of the mobile robot in hierarchical model," in Proc. IEEE Int. Conf. Netw., Sens. Control, Taipei, Taiwan, Mar. 2004, vol. 2, pp. 713-718.

[32] T. Dierks and S. Jagannathan, "Control of nonholonomic mobile robot formations: Backstepping kinematics into dynamics," in Proc. IEEE Int. Conf. Control Appl., Singapore, Oct. 2007, pp. 94-99.

[33] V. Boquete, R. Garcia, R. Barea, and M. Mazo, "Neural control of the movements of a wheelchair," J. Intell. Robot. Syst., vol. 25, no. 3, pp. 213 226, Jul. 1999.

[34] G. N. Marichal, L. Acosta, L. Moreno, J. A. Méndez, J. J. Rodrigo, and M. Sigut, "Obstacle avoidance for a mobile robot: A neuro-fuzzy approach," Fuzzy Sets Syst., vol. 124, no. 2, pp. 171-179, Dec. 2001.

[35] S. X. Yang and T. Hu, "An efficient neural network approach to real-time control of a mobile robot with unknown dynamics," Differ. Equ. Dyn. Syst., vol. 10, no. 1-2, pp. 151-168, 2002.

[36] A. Ollero, A. G. Gerezo, and J. V. Martinez, "Fuzzy supervisory path tracking of mobile robots," Control Eng. Practice, vol. 2, no. 2, pp. $313-$ 319, Apr. 1994.

[37] J. Ye, "Tracking control for nonholonomic mobile robots: Integrating the analog neural network into the backstepping technique," Neurocomputing, vol. 71, no. 16-18, pp. 3373-3378, Oct. 2008.

[38] T. Dierks and S. Jagannathan, "Asymptotic adaptive neural network tracking control of nonholonomic mobile robot formations," J. Intell. Robot Syst., Theory Appl., vol. 56, no. 1/2, pp. 153-176, 2009.

[39] A. L. Hodgkin and A. F. Huxley, "A quantitative description of membrane current and its application to conduction and excitation in nerve," $J$. Physiol., vol. 117, no. 4, pp. 500-554, 1952.

[40] H. Li and S. X. Yang, "A behavior-based mobile robot with a visual landmark recognition system," IEEE/ASME Trans. Mechatronics, vol. 8 , no. 3, pp. 390-400, Sep. 2003.

[41] S. X. Yang, "Neural dynamics and computation for real-time map building and path planning of mobile robots," Dyn. Continuous, Discrete Impulse Syst., Ser. B, vol. 10, no. 1, pp. 1-17, Jan. 2000.

[42] X. Wang, S. X. Yang, and M. Q.-H. Meng, "A co-evolution approach to sensor placement and control design for robot obstacle avoidance," Int. J. Inf. Acquisition, vol. 2, no. 2, pp. 77-91, Jun. 2005.

[43] C. Luo and S. X. Yang, "A bioinspired neural network for real-time concurrent map building and complete coverage robot navigation in unknown environments," IEEE Trans. Neural Netw., vol. 19, no. 7, pp. 1279-1298, Jul. 2008.

[44] G. Dissanayake, P. Newman, H. F. Durrant-Whyte, S. Clark, and M. Csobra, "A solution to the simultaneous localisation and mapping (slam) problem," IEEE Trans. Robot. Autom., vol. 17, no. 3, pp. 229-241, Jul. 2001.

[45] A. Zhu and S. X. Yang, "Neurofuzzy-based approach to mobile robot navigation in unknown environments," IEEE Trans. Syst., Man, Cybern. C, Appl. Rev., vol. 37, no. 4, pp. 610-621, Jul. 2007.

[46] A. L. Hodgkin, The Conduction of the Nervous Impulse. Liverpool, U.K.: Liverpool Univ., 1964.

[47] S. Grossberg, "Nonlinear neural networks: Principles, mechanisms, and architecture," Neural Netw., vol. 1, no. 1, pp. 17-61, 1988.

[48] H. Öğmen and S. Gagné, "Neural network architectures for motion perception and elementary motion detection in the fly visual system," Neural Netw., vol. 3, no. 5, pp. 487-505, 1990.

[49] M. A. Cohen and S. Grossberg, "Neural dynamic absolute stability of global pattern formation and parallel memory storage by competitive neural networks," IEEE Trans. Syst., Man, Cybern., vol. SMC-13, no. 5, pp. $815-826,1983$

[50] S. X. Yang and M. Meng, "An efficient neural network approach to dynamic robot motion planning," Neural Netw., vol. 13, no. 2, pp. 143-148, 2000

[51] S. X. Yang and M. Meng, "An efficient neural network method for real time motion planning with safety consideration," Robot. Auton. Syst., vol. 32 , no. $2 / 3$, pp. $115-128$, Aug. 2000. 
[52] S. X. Yang and M. Q.-H. Meng, "Neural network approaches to dynamic collision-free trajectory generation," IEEE Trans. Syst., Man, Cybern. B, Cybern., vol. 31, no. 3, pp. 302-318, Jun. 2001.

[53] S. X. Yang and M. Q.-H. Meng, "Real-time collision-free motion planning of a mobile robot using a neural dynamics-based approach," IEEE Trans. Neural Netw., vol. 14, no. 6, pp. 1541-1552, Nov. 2003.

[54] X. Yuan and S. X. Yang, "Virtual assembly with biologically inspired intelligence," IEEE Trans. Syst., Man, Cybern. C, Appl. Rev., vol. 33, no. 2, pp. 159-167, May 2003.

[55] X. Yuan and S. X. Yang, "Multi-robot-based nanoassembly planning with automated path generation," IEEE/ASME Trans. Mechatronics, vol. 12, no. 3, pp. 352-356, Jun. 2007.

[56] S. X. Yang and C. Luo, "A neural network approach to complete coverage path planning," IEEE Trans. Syst., Man, Cybern. B, Cybern., vol. 34, no. 1, pp. 718-725, Feb. 2004.

[57] A. R. Willms and S. X. Yang, "An efficient dynamic system for real-time robot path planning," IEEE Trans. Syst., Man, Cybern. B, Cybern., vol. 36, no. 4, pp. 755-766, Aug. 2006.

[58] A. R. Willms and S. X. Yang, "Real-time robot path planning via a distance-propagating dynamic system with obstacle clearance," IEEE Trans. Syst., Man, Cybern. B, Cybern., vol. 38, no. 3, pp. 884-893, Jun. 2008.

[59] H. Qu, S. X. Yang, A. R. Willms, and Z. Yi, "Real-time robot path planning based on a modified pulse coupled neural network model," IEEE Trans. Neural Netw., vol. 20, no. 11, pp. 1724-1739, Nov. 2009.

[60] K. C. Koh and H. S. Cho, "A smooth path tracking algorithm for wheeled mobile robots with dynamic constraints," J. Intell. Robot. Syst., Theory Appl., vol. 24, no. 4, pp. 367-385, Apr. 1999.

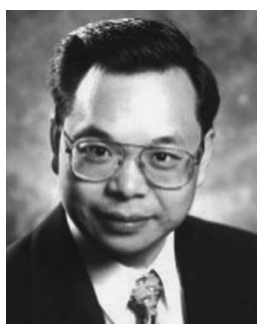

Simon X. Yang (S'97-M'99-SM'08) received the B.Sc. degree in engineering physics from Beijing University, Beijing, China, in 1987, the first of two M.Sc. degrees in biophysics from the Chinese Academy of Sciences, Beijing, in 1990, the second M.Sc. degree in electrical engineering from the University of Houston, Houston, TX, in 1996, and the Ph.D. degree in electrical and computer engineering from the University of Alberta, Edmonton, AB, Canada, in 1999.

He is currently a Professor and the Head of the Advanced Robotics and Intelligent Systems Laboratory at the University of Guelph, Guelph, ON, Canada. His research interests include intelligent systems, robotics, sensors and multisensor fusion, wireless sensor networks, control systems, and computational neuroscience.

Dr. Yang serves as an Associate Editor of the IEEE TRANSACTIONS ON Neural Networks, the IEEE Transactions on Systems, Man, AND CYBERNETICS-PART B, and several other journals. He has been involved in the organization of many conferences.

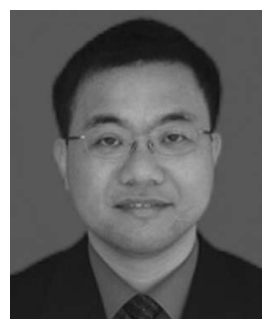

Anmin Zhu (S'04-M'09) received the B.Sc. and M.Sc. degrees from Tongji University, Shanghai, China, in 1987 and 1990, respectively, and the Ph.D. degree from the University of Guelph, Guelph, ON, Canada, in 2005.

$\mathrm{He}$ is currently a Faculty Member with the College of Electrical and Information Engineering, Shenzhen University, Shenzhen, China. He is also currently with the Advanced Robotics and Intelligent Systems Laboratory, University of Guelph. His research interests include fuzzy systems, neural networks, robotics, self-organizing arrangement, machine intelligence, multiagent systems, and control systems.

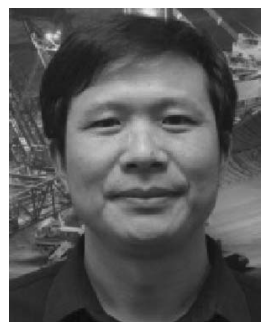

Guangfeng Yuan received the B.Sc. degree from Jiangsu University, Zhenjiang, China, in 1995, and the M.Sc. degree from the University of Guelph, Guelph, ON, Canada, in 2001.

He is currently a Project Engineer with the Electrical Department, Detroit Heavy Truck Engineering, Novi, MI. He is also currently with the Advanced Robotics and Intelligent Systems Laboratory, University of Guelph. His research interests include hydroelectric control, motion control, machine automatic control systems, remote communication, and

robotics.

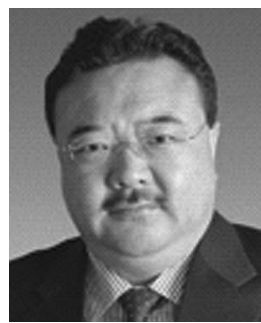

Max Q.-H. Meng (F'08) received the Ph.D. degree in electrical and computer engineering from the University of Victoria, Victoria, BC, Canada, in 1992, following the M.S. degree from the Beijing Institute of Technology, Beijing, China, in 1988.

$\mathrm{He}$ has been a Professor of Electronic Engineering with the Chinese University of Hong Kong (CUHK), Hong Kong, China, since 2002, after working for ten years with the Department of Electrical and Computer Engineering, University of Alberta, Edmonton, $\mathrm{AB}$, Canada. He is jointly appointed as the Director of the Center for Intelligent Sensing at the Chinese Academy of Sciences (CAS)/CUHK Shenzhen Institute of Advanced Integration Technology and as an Overseas Outstanding Scholar Chair Professor of the CAS. His research interests include robotics and active medical devices, biosensors and sensor networks, network-enabled systems, and intelligent systems.

Dr. Meng has served as an Editor of the IEEE/ASME TRANSACTIONS ON MECHATRONICS and as an Associate Editor of the IEEE TRANSACTIONS ON FUZZY Systems. He was a recipient of the IEEE Third Millennium Medal award. 